\title{
Automated comparative sequence analysis identifies mutations in $89 \%$ of NFl patients and confirms a mutation cluster in exons 11-17 distinct from the GAP related domain
}

\author{
C Mattocks, D Baralle, P Tarpey, C ffrench-Constant, M Bobrow, J Whittaker
}

J Med Genet 2004;41:e48 (http://www.jmedgenet.com/cgi/content/full/41/4/e48). doi: 10.1136/jmg.2003.011890

$\mathrm{N}$ eurofibromatosis type 1 (NF1), formerly known as Von Recklinghausen Neurofibromatosis, is a common genetic disorder affecting approximately 1 in 30005000 people. It is a fully penetrant autosomal dominant disorder. Strict diagnostic criteria that include café au lait spots, neurofibromas, plexiform neurofibromas, freckling in the axillary or inguinal regions, Lisch nodules (iris haematomas), optic or chiasma glioma, pseudoarthrosis, and sphenoid dysplasia define NFl. Most disease features are present in more than $90 \%$ of patients at puberty. ${ }^{1}$ Further manifestations are known to occur in this disorder, including macrocephaly, short stature, learning difficulties, scoliosis and certain malignancies..$^{2-4}$ There is, however, great intra and interfamilial phenotypic variability. In addition a number of patients who have a clinical picture suspected to be NFl do not fulfil the diagnostic criteria particularly in the younger age groups. As a consequence genetic testing would have a major impact on the diagnosis and management of these families.

The NFl gene maps to chromosome 17q11.2 and is thought to be a tumour suppressor gene because loss of heterozygosity is associated with the occurrence of benign and malignant tumours in tissues derived from the neural crest $^{5-7}$ as well as myeloid malignancies. ${ }^{8}$ It spans a region of about $350 \mathrm{~kb}$ of genomic DNA and contains 60 exons. ${ }^{9-11}$ It harbours at least three other embedded genes-EV12A, EV12B, and ONGPtranscribed from the opposite strand of NFl intron 27b. The NFl gene transcribes several mRNAs in the size range 11$13 \mathrm{~kb}$ expressed in neurones, oligodendrocytes and nonmyelinating Schwann cells. ${ }^{12}$ The most common transcript codes for a polypeptide of 2818 amino acids called neurofibromin. ${ }^{13-15}$ A 360 amino acid region of the predicted protein product shows homology with the GTPase activating (GAP) family of proteins in yeast and mammals. ${ }^{16}$ The GAP related domain (GRD) is the only known functional domain of the NFl gene and spans exons 20-27a (bases 3497-4661). It has been found to interact with p21 ras ${ }^{17}$ and GTP, and is thought to prevent uncontrolled cell proliferation by converting active RasGTP to inactive RasGDP. In keeping with this, RasGTP levels may be elevated in human NFl peripheral nerve tumours in which neurofibromin is reduced or absent. ${ }^{18}$ This region of the gene has also been reported as representing a "hot spot" for mutations consistent with an important functional role. ${ }^{19}$ More recently, it has also been shown that learning deficiencies associated with NFl may be caused by excessive Ras activity, which leads to impairments in long term potentiation caused by increased GABA mediated inhibition. ${ }^{20} \mathrm{~A}$ putative second hot spot in a possible functional domain upstream of GRD, comprising exons 1117, has been described. ${ }^{21}$ The region encodes a domain rich in cysteine and serine, with three cysteine pairs suggestive of ATP binding, as well as three potential cAMP dependent protein kinase recognition sites. Further evidence for cAMP

\section{Key points}

- Neurofibromatosis type 1 (NF1) is a common autosomal dominant genetic disorder caused by mutations in the NFl gene. Mutation detection in NF1 has been a major challenge due to the large size of the gene and lack of mutational hotspots.

- We report here a study on 91 subjects fulfilling the National Institutes of Health (NIH) NFl diagnostic criteria, in which we achieve a mutation detection rate of $89 \%$ using automated comparative sequence analysis (ACSA). This detection rate is the highest for a single technique and is therefore appropriate for routine clinical practice.

- Our data show that mutations are evenly distributed along the coding sequence of the NFl gene and not clustered at the GAP-related domain (GRD). However, we confirm the presence of a second putative functional domain upstream of the GRD at exons 11-17.

signalling being perturbed in NFl comes from mutations in Drosophila neurofibromin that have been shown to inhibit the CAMP and protein kinase signalling pathway.

Mutation detection in NFl has been made difficult by the large size of the gene, the existence of a number of homologous pseudogene sequences spread throughout the genome, and the lack of defined mutational hotspots. To overcome these problems, a variety of techniques have been employed for screening the NFl gene. Most studies have been based on single strand conformation polymorphism, heteroduplex analysis, temperature gradient gel electrophoresis and denaturing gradient gel electrophoresis. In the largest study to date, involving 500 patients, Fahsold et $\mathrm{al}^{21}$ used a protein truncation test, temperature gradient gel electrophoresis, and direct genomic sequencing to examine all of the individual exons, finding sequence variants in 301 patients. Within these variants 278 mutations were considered pathogenic. In two more papers published recently, the methodologies were used sequentially to raise mutation detection rates. In the study of Ars et $\mathrm{al}^{22}$ on 80 patients, using cDNA single strand conformation polymorphism and heteroduplex analysis, a detection rate of $70-80 \%$ of mutations was achieved. Messiaen et $\mathrm{al}^{23}$ used a protein truncation test, fluorescence in situ hybridisation, southern blot and cytogenetic analysis

Abbreviations: ACSA, automated comparative sequence analysis; CSA, comparative sequence analysis; GRD, GAP-related domain; NF1, neurofibromatosis type 1; NIH, National Institutes of Health 
to study 67 patients, and reported a detection rate of $95 \%$, including a high frequency of unusual splicing defects. The sensitivity of each individual technique is hard to establish, as mutation analysis reports have either concentrated on groups of exons, included small numbers in their studies, or used a combination of techniques. In reviews of known NFl mutations, ${ }^{1}$ several mutation types are described, but no correlation with phenotype was documented. Most of the fully characterised NFl mutations are either nonsense or frameshift mutations, which presumably lead to premature truncation of neurofibromin. Large deletions of the NFI gene are thought to account for less than $10 \%$ of cases. A relationship between whole gene deletions and a more severe NF1 phenotype has been reported.

Although the application of these different techniques allows a clinically useful mutation detection rate, the extremely time consuming nature of the work makes them impractical for routine clinical work. An alternative strategy based on automated sequencing techniques would clearly be a more sensitive and informative method for mutation testing. However these techniques are not widely used as a first screen since analysis of the results can be both cumbersome and time consuming, and with current methodologies raw sequence data often require extensive manipulation before analysis. We have previously described a novel analytical approach, comparative sequence analysis (CSA), to overcome this problem. ${ }^{24}$ This technique allows automation of data analysis, with accurate analyses of 96 lanes, and is therefore achievable in 1-2 minutes. Complete processing of four 96 well plates is therefore now feasible for a high throughput team in a single day. It is particularly suited to the scanning of large genes, with large numbers of different mutations, which are expected to be in heterozygous form. Here we apply this technique to the problem of NFI gene mutations.

\section{MATERIALS AND METHODS Subjects}

One hundred and sixty seven subjects were screened in three batches designated D02, D04, and D05. These included 131 unrelated patients suspected of having NF1, 24 relatives of the probands, and 12 normal controls. All subjects were assessed using the criteria of the National Institutes of Health $(\mathrm{NIH})^{25}$ to determine NFl status. Ninety one unrelated subjects fulfilled the NIH criteria for NFl and were categorised as definitely affected. Thirteen unrelated subjects fell marginally outside the NIH criteria and were categorised as possibly affected. The remaining 27 unrelated patients were classified as unaffected.

\section{Polymerase chain reaction}

Genomic DNA was extracted from peripheral blood using the Nucleon BACC2 kit and resuspended in TE buffer to a concentration of approximately $500 \mu \mathrm{g} / \mathrm{ml}$. The entire reading frame of the NFI gene was PCR amplified exon by exon, in a total of 58 amplicons. In general primers were designed so that each amplicon comprised an exon flanked by at least 50 base pairs of intron on either side. One primer in each pair was tailed with an M13 primer sequence (M13F, TGTAAAACGACGGCCAGT or MI3R, CAGGAAACA GCTAT GACC) to provide a binding site for M13 universal sequencing primer. Care was taken to ensure any repetitive sequences flanking the exon lay at the opposite end of the amplicon with respect to the M13 sequencing primer sequence. In general the PCR temperature profile was: initial denaturation at $95^{\circ} \mathrm{C}$ for $2 \mathrm{~min}$, followed by 30 cycles with annealing for $30 \mathrm{~s}$, elongation at $72^{\circ} \mathrm{C}$ for $1 \mathrm{~min}$, and denaturation at $95^{\circ} \mathrm{C}$ for $30 \mathrm{~s}$. Primer sequences, annealing temperatures and buffers used are given in Table 1.

\section{Sequencing}

Polymerase chain reaction products were purified by adding $1 \mathrm{U}$ shrimp alkaline phosphatase (Amersham) and $10 \mathrm{U}$ exonuclease I (NEB) to each reaction and incubating at $37^{\circ} \mathrm{C}$ for $30 \mathrm{~min}$ followed by $95^{\circ} \mathrm{C}$ for $10 \mathrm{~min}$ to denature the enzymes. Purified PCR products were then sequenced in a single orientation dependant on the location of the M13 tail on the PCR primers. Reaction mixtures comprised $2 \mu \mathrm{l}$ of PCR product, $0.7 \mu \mathrm{l}$ BigDye $\mathrm{V}$ sequencing reaction mix (Applied Biosystems), $1.05 \mu \mathrm{l} 5 \times$ sequencing diluent (Applied Biosystems), $0.35 \mu \mathrm{l}$ primer at $10 \mathrm{pmol} / \mu \mathrm{l}, 5 \%(\mathrm{v} / \mathrm{v})$ betaine (Sigma) and $\mathrm{ddH}_{2} \mathrm{O}$ to $7 \mu \mathrm{l}$. Initial denaturation was at $95^{\circ} \mathrm{C}$ for $5 \mathrm{~min}$, followed by 25 cycles with annealing at $50^{\circ} \mathrm{C}$ for $15 \mathrm{~s}$, elongation at $60^{\circ} \mathrm{C}$ for $4 \mathrm{~min}$, and denaturation at $95^{\circ} \mathrm{C}$ for $30 \mathrm{~s}$. For batches D02 and D04, completed sequencing reactions were purified using Dynapure ${ }^{\mathrm{TM}}$ Dye Terminator Removal Ver.2 (Dynal AS, Oslo, Norway) according to the manufacturer's protocol. Products were resuspended in $1.5 \mu \mathrm{l}$ formamide and $0.8 \mu \mathrm{l}$ dotted on 96 well RapidLoad ${ }^{\mathrm{TM}}$ membrane combs (Web Scientific Crewe, UK). The ABI 377 was loaded using the "water protocol" as described by Web Scientific. For batch D05, four plates of sequencing reactions were transferred to a single Multiscreen 384 well filter plate (Millipore, Bedford, UK) and purified according to the manufacturer's protocol. The samples were then resuspended in $15 \mu \mathrm{l} \mathrm{Hi-Di} \mathrm{formamide} \mathrm{(Applied} \mathrm{Biosystems)} \mathrm{with} 0.05 \mu \mathrm{l}$ GeneScan $^{\mathrm{TM}} 500$ LIZ size standard (Applied Biosystems) for each reaction and transferred to a 384 MicroAmp optical plate (Applied Biosystems) for loading on the ABI 3100.

\section{Analysis}

Analysis was carried out using an automated comparative sequence analysis (CSA) program, AutoCSA12. This will be described fully elsewhere. In brief, the technique involves horizontal alignment of each of the four base traces separately with a control trace. The program then calculates peak position and height for each base and will highlight all differences. No electrophoretograms were examined directly unless variations were called by the program. Called variations were examined in GeneScan V3 (Applied Biosystems) by non-automated CSA. ${ }^{24}$ For batches D02 and D04, horizontal alignment was achieved by generating an artificial size standard that applied arbitrary numbers to the peaks in one of the four traces (usually the red C trace). Batch D05 was horizontally aligned using the GeneScan $^{\mathrm{TM}} 500$ LIZ size standard. Sequence variations were confirmed by resequencing using a new aliquot of DNA. Fig 1 shows an example of CSA.

\section{RESULTS}

We have studied 131 unrelated subjects referred for NFl screening. Of the 91 subjects who fulfilled the NIH criteria for NFl we found putative mutations in 81 subjects $(89.0 \%)$. In the possibly affected group of 13 patients we found putative mutations in 8 patients (61.5\%). No mutations were found in any of the patients classified as unaffected. Thus, of the 104 patients with possible or definite NFI we detected a total of 89 putative mutations (85.6\%). Altogether, 77 different potentially significant sequence variations were found, of which 57 (74\%) were novel. Sequence variants were classified as pathogenic mutations if they fulfilled one of the following criteria: a) they resulted in the introduction of a stop codon that truncated the protein-for example, frame shifts - or an amino acid alteration to a stop codon, b) they affected an invariant splice consensus sequence, c) they could be demonstrated to be de novo in the patient (that is, not present in the parents), d) they were previously reported as pathogenic in the literature and fulfilling any of the above or with supporting data to show that function was impaired. Table 2 provides a summary of the NF1 mutations and sequence variations found. 
Table 1 Primer sequences, annealing temperatures, and buffers used for PCR reactions

\begin{tabular}{|c|c|c|c|c|c|c|c|c|c|}
\hline \multirow{2}{*}{$\begin{array}{l}\text { Exon } \\
1\end{array}$} & \multicolumn{2}{|c|}{ Forward primer } & \multicolumn{2}{|c|}{ Reverse primer } & Length & $T_{a}$ & \multicolumn{2}{|r|}{ Sequence } & \multirow{2}{*}{$\begin{array}{l}\text { Orientation } \\
\text { Reverse }\end{array}$} \\
\hline & IF.A & AGACCCTCTCCTTGCCTCTT & IR.0 & M13RX-ATGGAGGGTCGGAGGCTG & 458 & 55.0 & $\mathrm{D}$ & M13R & \\
\hline 2 & $2 F .1$ & TGGCAAGTAAGTTATTATGGTC & $2 R .1$ & MI 3F-AATCAAAAAGAAAAGAAAGCAA & 288 & 55.0 & B & MI3F & Reverse \\
\hline 3 & $3 F .1$ & GTITGCCTIAGACTITAGTITI & 3R. 1 & M13F-CATCTGTACTTTGGGACATAA & 368 & 55.0 & B & M13F & Reverse \\
\hline $4 a$ & $4 \mathrm{aF} .0$ & TTTGAAAATTTTCATAATAGAAAATGT & 4aR.0 & M13RX-GGTCAAAGCTGCTGTGAG & 435 & 55.0 & $\mathrm{C}$ & M13R & Reverse \\
\hline $4 b$ & 4bF.0 & M13FX-TCCTGGCCTCAAGTGGTC & 4bR.0 & M13RX-TTATAAAANCCAGATTGGTGTTC & 386 & 57.0 & B & M13F & Forward \\
\hline $4 c$ & $4 \mathrm{cF} .0$ & MI3FX-TCCTAGCAGACAACTATCGA & $4 c R .0$ & AAAAAAAAATCAATCGTATCCTTA & 582 & 55.0 & C & M13F & Forward \\
\hline 6 & $6 \mathrm{~F} .0$ & M13FX-CATGTTIATCTTITAAAAATGTTGCC & $6 R .0$ & ATAATGGAAATAATTTTGCCCTCC & 323 & 55.0 & C & M13F & Forward \\
\hline 7 & 7F.0 & MI3FX-TGCTATAATATTAGCTACATCTGG & 7R.0 & CCTATGAACTTATCAACGAAGAG & 395 & 55.0 & B & M13F & Forward \\
\hline 8 & $8 \mathrm{~F} .0$ & M13FX-TTGTGTGGGTAATGTGTTGA & $8 R .0$ & AAATATAGTTAGATAAAAACCAATG & 270 & 55.0 & $\mathrm{~B}$ & M13F & Forward \\
\hline 9 & 9F.1 & TATTGGTGTTCTITTGG & 9R. 1 & MI3F-AATTTAGCAATACCTITTGG & 204 & 55.0 & B & M13F & Reverse \\
\hline $9 a$ & $9 \mathrm{aF} .0$ & TCCGCTGTGGCTCAGAACAC & 9aR.0 & M13RX-AGTAGAAGAGGATGCACAGCC & 315 & 60.0 & B & M13R & Reverse \\
\hline $10 a$ & 10aF.0 & MI3FX-GATAAACAGAGCATACAACTCA & 10aR.0 & 0 AAATGCAATAGAAAGGAGGTGA & 270 & 55.0 & B & M13F & Forward \\
\hline $10 \mathrm{~b}$ & 10bF.A & A AACTATTGAGTGTTCTACTATACC & 10bR.0 & O MI3RX-TTGGCGATTCAGCTAAACC & 374 & 55.0 & G & $M 13 R$ & Reverse \\
\hline $10 c$ & 10cF.1 & M13F-CGTCCAGCCTAGTTCTAGAA & 10cR. 1 & АССССТTСТTТСТССАТ & 444 & 55.0 & C & M13F & Forward \\
\hline 11 & $11 \mathrm{~F} .0$ & MI3FX-ATAAGTACTCCAGTGTTATGT & $11 R .0$ & TAAAGTTGAAATTIAAAAAATTAAAGTAC & 179 & 52.0 & $\mathrm{C}$ & M13F & Forward \\
\hline $12 a$ & $12 \mathrm{aF} .0$ & $\begin{array}{l}\text { MI3FX- } \\
\text { AAACCTTACAAGAAAAACTAAGCT }\end{array}$ & $12 \mathrm{aR} .0$ & O АТТАССАТТССАААТАТТСТTССА & 325 & 55.0 & $\mathrm{C}$ & $\mathrm{M} 13 \mathrm{~F}$ & Forward \\
\hline $12 b$ & $12 \mathrm{bF} .0$ & MI3FX-TTTCTAGTAAATCTCCTСAAGT & $12 b R .0$ & O AT & 404 & 55.0 & $\mathrm{C}$ & $113 \mathrm{~F}$ & orward \\
\hline 13 & $13 \mathrm{~F} .1$ & AAGATATITTGGGGTTTGAA & $13 R .1$ & M13R-GCCATGTGCTTTGAGG & 508 & 55.0 & $\mathrm{~B}$ & M13R & \\
\hline 14 & $14 \mathrm{~F} .0$ & MI3FX-AGCTTATCAGGTTCTCCATTGG & $15 R .0$ & $\begin{array}{l}\text { M13R- } \\
\text { AGTTAACAGACAAAAGTCAACTTTACAG }\end{array}$ & 535 & 55.0 & D & MI3F & Forward \\
\hline 15 & $14 \mathrm{~F} .0$ & M13FX-AGCTTATCAGGTTCTCCATTGG & $15 R .0$ & $\begin{array}{l}\text { M13R- } \\
\text { AGTTAACAGACAAAAGTCAACTTTACAG }\end{array}$ & 535 & 55.0 & $\mathrm{D}$ & M13R & Reverse \\
\hline 16 & $16 \mathrm{~F} .0$ & MI3FX-TGGATAAAGCATAAT & $16 \mathrm{~F}$ & TAGAGAAAGGTGAAAAATAAGAG & 571 & 55.0 & B & $113 \mathrm{~F}$ & Forward \\
\hline 17 & $17 \mathrm{~F} .0$ & CTGTGTGT & 17R.0 & ATCAATTACTACCAGTATCAG & 339 & 55.0 & $\mathrm{~B}$ & & \\
\hline 18 & $18 \mathrm{~F} .0$ & AGAAGTTGTGTACGTTCTITTCT & 18R.0 & TACCAATAACCGC & 388 & 0 & I & & \\
\hline $19 a$ & $19 \mathrm{aF} .0$ & MI3FX-ATGTCACTTAGGTTATCTGG & 19aR.0 & GTTATAACTCTC & 290 & 60.0 & B & & \\
\hline $19 \mathrm{~b}$ & 19bF.1 & M13F-ATTTGAGGGGAAGTGAA & $19 \mathrm{bR} .2$ & CITGCT & 312 & 55.0 & $\mathrm{E}$ & MI3R & \\
\hline 20 & $20 \mathrm{~F} .0$ & $\begin{array}{l}\text { MI3FX- } \\
\text { CATTCACACCATGCACATATGATTG }\end{array}$ & $21 R .0$ & MI3R-GATTTGCTATGTGCCAGGCAC & 703 & 55.0 & $\mathrm{E}$ & MI3F & Forward \\
\hline 21 & $20 \mathrm{~F} .0$ & $\begin{array}{l}\text { M13FX- } \\
\text { CATTCACACCATGCACATATGATTG }\end{array}$ & $21 R .0$ & M13R-GATTTGCTATGTGCCAGGCAC & 703 & 55.0 & $E$ & M13R & Reverse \\
\hline 22 & $22 \mathrm{~F} .0$ & MI3FX-TGCTACTCTTTAGCTTCCTAC & $22 R .0$ & ACAGCGGT & 537 & 55.0 & B & M13F & Forward \\
\hline 23-2 & $\begin{array}{l}23- \\
2 F .0\end{array}$ & M13FX-CTTAATGTCTGTATAAGAGTCTC & $\begin{array}{l}23- \\
2 R .0\end{array}$ & $\mathrm{ACT}$ & 290 & 55.0 & C & M13F & \\
\hline $23 a$ & $23 \mathrm{aF} .0$ & & $23 a R .0$ & GA & 465 & 55.0 & 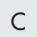 & $M 13 R$ & \\
\hline 24 & $24 \mathrm{~F} .0$ & M13FX-TTGAACTCTITIGTTITCATGTCTT & $24 R .0$ & GGAATTTAAGATAGCTAGATTATC & 289 & 55.0 & $\mathrm{C}$ & M13F & Forward \\
\hline 25 & $25 \mathrm{~F} .0$ & $\begin{array}{l}\text { M13FX- } \\
\text { AATATAATAATTATATTTGGGAAGGT }\end{array}$ & $25 R .0$ & GAAAATATTTGATTCAAACAGAGC & 360 & 55.0 & $\mathrm{~B}$ & M13F & Fonward \\
\hline 26 & $26 \mathrm{~F} .1$ & MI3F-CCCTCCATATTTGTAATCTT & $26 R .1$ & & 324 & 58.0 & B & M13F & Forward \\
\hline $27 a$ & $27 \mathrm{aF} .1$ & AGGGCATTITAATCTITTAT & $27 \mathrm{aR} .1$ & MI3F-GCAAACTCTCCTTCTCAAC & 406 & 55.0 & $\mathrm{~F}$ & $\mathrm{M} 13 \mathrm{~F}$ & \\
\hline $27 b$ & $27 \mathrm{bF} .1$ & M13R-GCCAAATTACCCTITAGAATG & $27 b R .1$ & 1 AACCAAACTTGCCATCTCT & 348 & 55.0 & C & $M 13 R$ & Forward \\
\hline $28 F^{*}$ & $28 \mathrm{~F} .1$ & MI3F-GACTTTGAAGAATTGTITTATA & $28 R .0$ & MI3RX-CTATAGGGGTAGGACACC & 693 & 55.0 & $\mathrm{E}$ & M13F & \\
\hline $28 R^{*}$ & $28 \mathrm{~F} .1$ & M13 & $28 R .0$ & GACACC & 693 & 55.0 & $\mathrm{E}$ & $113 R$ & e \\
\hline $29 F^{*}$ & $29 \mathrm{~F} .1$ & TGGGAACTCTTCCTTA & $29 R .0$ & AACACTGCATACCTTCCAATT & 635 & 55.0 & $\mathrm{E}$ & $\mathrm{F}$ & ard \\
\hline $29 R^{*}$ & $29 \mathrm{~F} .1$ & M13F-TACAATGGTGGGAACTCTTCCTTA & $29 R .0$ & MI3RX-ACCAACACTGCATACCTTCCAATT & 635 & 55.0 & $\mathrm{E}$ & M13R & Reverse \\
\hline 30 & $30 F .0$ & GCCTCACAGTGCTCTTATGG & 3OR.0 & MI3RX-CCTTCAGAGTTCCTTCAGTIAC & 628 & 55.0 & $\mathrm{C}$ & M13R & Reverse \\
\hline 31 & $31 \mathrm{~F} .0$ & TGTTGATGTGATTTTCATTGACCA & $31 R .0$ & MI3RX-CCAATGTGGCACCAGATAAATAT & 337 & 55.0 & $\mathrm{~B}$ & MI3R & Reverse \\
\hline 32 & $32 \mathrm{~F} .0$ & M13FX-ATCTAGTATTITGGAGGCCTCAG & $32 R .0$ & CAGATATGCTATAGTACAGAAGG & 334 & 55.0 & $\mathrm{C}$ & $\mathrm{M} 13 \mathrm{~F}$ & Forward \\
\hline 33 & $33 \mathrm{~F} .0$ & M13FX-TATCTGTITTATCATCAGGAGG & $33 R .1$ & M13R-TAAAATGGAGAAAGGAACTGG & 497 & 55.0 & $E$ & $M 13 R$ & Reverse \\
\hline 34 & $34 \mathrm{~F} .1$ & MI3F-AAAATGAAACATGGAACTITAG & $34 R .0$ & $\begin{array}{l}\text { M13RX- } \\
\text { TAAGCATTAAGTACAAAATAGCAC }\end{array}$ & 439 & 55.0 & $\mathrm{E}$ & M13F & Forward \\
\hline 35 & $35 \mathrm{~F} .0$ & AT & $35 R .0$ & GAAAAATG & 45 & 55.0 & 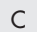 & $3 R$ & \\
\hline 37 & $37 F .0$ & M13FX-ATTCCGAGATTCAGTTTAGGAG & $37 R .0$ & AGTAACATTCAACACTGATACCC & 256 & 55.0 & $\Pi$ & MI3F & ward \\
\hline 38 & $38 \mathrm{~F} .1$ & ACATGGGTAATTIAGGAAGATAAG & $38 R .1$ & MI3F-CAACAAGAAAAGATGGAAGA & 335 & 55.0 & C & $\mathrm{M} 13 \mathrm{~F}$ & Reverse \\
\hline 39 & 39F.0 & CTACTGTGTGAACCTCATCAACC & 39R.0 & $\begin{array}{l}\text { M13RX- } \\
\text { GTAAGACATAAGGGCTAACTTACTTC }\end{array}$ & 306 & 55.0 & I & M13R & Reverse \\
\hline 40 & 40F.0 & GAAGACCTCAGCAGAT & 4OR.0 & M13RX-AACTTTCTGCTCTGCCACGCA & 345 & 55.0 & 1 & MI3R & \\
\hline 41 & 41F.0 & TTCATCCTGIITAAAGTCACACTTG & $41 R .0$ & M13RX-TTGCCTCCATTAGTTGGAAAATTG & 295 & 55.0 & $\mathrm{H}$ & $M 13 R$ & Reverse \\
\hline 42 & $42 \mathrm{~F} .0$ & GAAGGAGCAAACGATGGTTG & $42 R .0$ & M13RX-AAACTTTGCTACACTGACATGG & 371 & 55.0 & $\mathrm{C}$ & MI3R & Reverse \\
\hline 43 & 43F.0 & GTATTAGAGCTITCTITGAGTC & 43R.0 & M13RX-GAATGGTAGAGTITATCTITTG & 421 & 55.0 & $\mathrm{C}$ & $M 13 R$ & Reverse \\
\hline 44 & $44 F .2$ & M13R-GGTGAAGTGATTATCCAGGTC & $44 R .2$ & TGTIATCTGTITGGGTGAGAAG & 328 & 55.0 & $E$ & $M 13 R$ & Forward \\
\hline 45 & $45 \mathrm{~F} .0$ & MI3R-AGATAACAATTCAGCCACAAAG & $45 R .0$ & TAAAGACAGGCACGAAGGT & 225 & 55.0 & $\mathrm{~F}$ & MI3R & Forward \\
\hline 46 & 46F. 1 & M13R-ATTTTGGCACATTATTCTGG & 46R. 1 & GCGCATGTTAGCAAGTT & 316 & 55.0 & C & MI3R & Forward \\
\hline 47 & 47F.0 & CTGTTACAATTAAAAGATACCTTGC & 47R.0 & M13RX-GTGTTCTTAAAGCAGGCATAC & 204 & 55.0 & $\mathrm{H}$ & M13R & Reverse \\
\hline 48 & $48 \mathrm{~F} .0$ & TITTGGCTTCAGATGGGGATITAC & $48 R .0$ & MI3RX-AAGGGAATTCCTAATGTTGGTGTC & 373 & 55.0 & $\mathrm{~B}$ & $M 13 R$ & Reverse \\
\hline $48 a$ & $48 \mathrm{aF} .0$ & ATCTAGTATCTAATTGTATTTCACCC & $48 a R .0$ & O MI3RX-AGACTGAGCTTACAGGGAC & 265 & 55.0 & A & $M 13 R$ & Reverse \\
\hline 49 & 49F.0 & M13FX-CTGGGAGAAACAGGCTATAC & $49 R .0$ & CACTITCTTTGCAGTTGTTCTG & 639 & 58.0 & $\mathrm{E}$ & M13F & Forward \\
\hline
\end{tabular}

M13F, TGTAAAACGACGGCCAGT; M13FX, GTGGTGTAAAACGACGGCCAGT; M13R, CAGGAAACAGCTATGACC; M13RX, GTGGCAGGAAACAGCTATGACC

* PCR product sequenced in both directions for single coverage

\section{Mutation spectrum}

By far the largest group of variations were single base pair substitutions, which were detected in 61 cases $(67 \%)$. Of these, $43(72 \%)$ were transitions and $17(28 \%)$ were transversions, proportions corresponding closely with those observed by Ars et al. ${ }^{22}$ Mutation at $\mathrm{CpG}$ dinucleotides account for $20 / 60$ (33\%) of the single base pair substitutions. Of these single base pair substitutions, the most frequently detected single group of sequence variants was that of missense substitutions, which were found in 25 subjects 


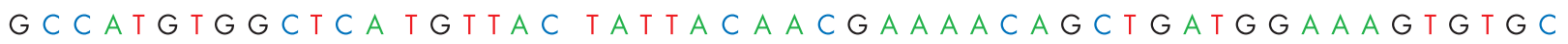

90 100
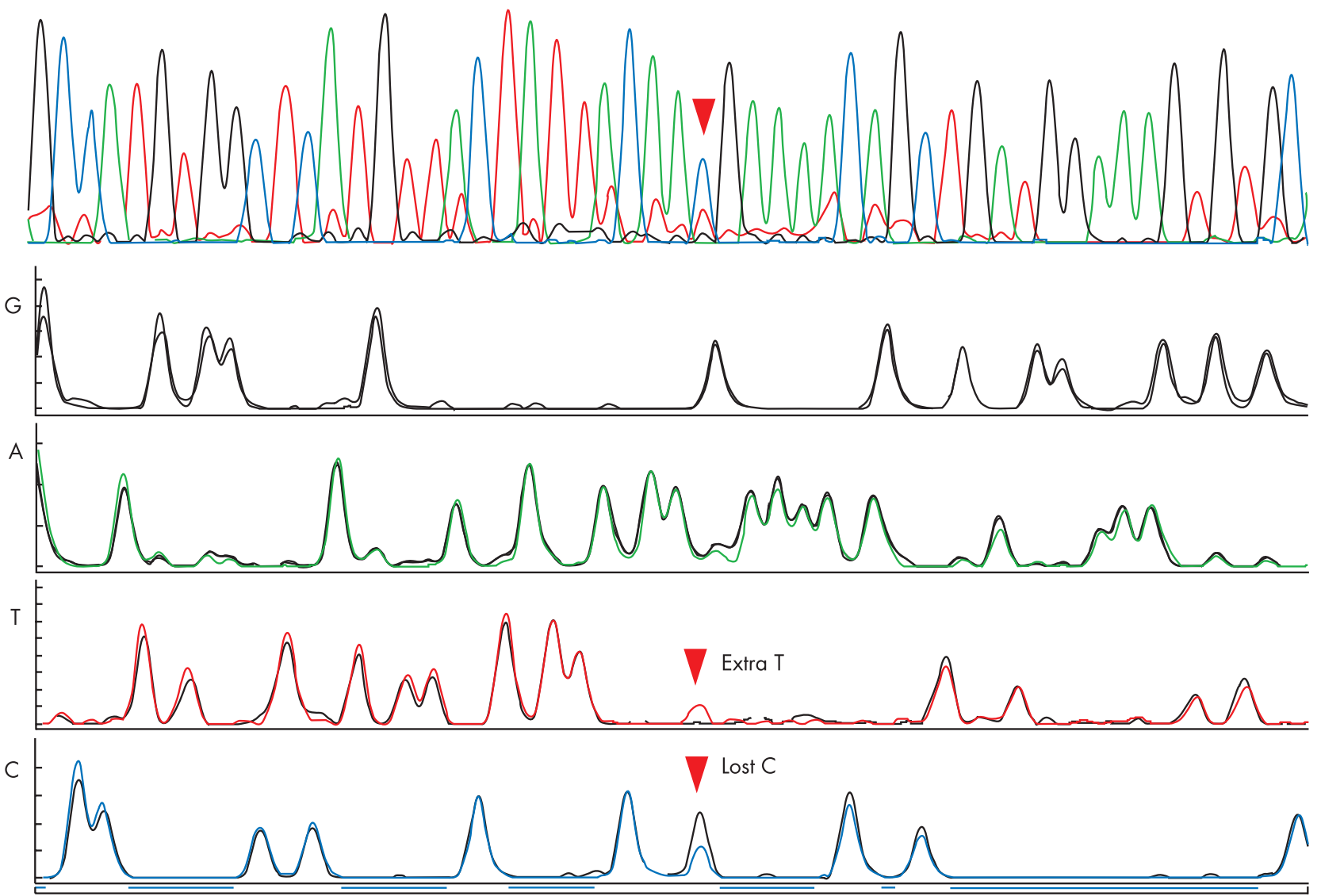

Figure 1 Example of mutation detected by CSA. Normal sequence indicated in black, test sample indicated in colour. The base represented by each track is indicated in the top left of each panel. A heterozygote point mutation is seen $\mathrm{C}>\mathrm{T}$. The half height peak in the $\mathrm{C}$ trace and the extra peak in the T trace can be easily seen. These differences can be read by the software program ACSA.

$(28 \%)$. Of these, 18 were found in patients who fulfilled the NIH criteria while seven were found in possibly affected patients. Only five of these have been previously described but a further 11 have closely related variations (affecting the same codon) in the literature. There were two mutations of note. First, the variant of codon 844 (2531T >C L844P), which was found twice in this study, and is one of at least four known variations of this codon, which was found in conjunction with a second variant affecting codon 838 (2514 C > G I838M). It is not known if these two variants occurred in trans or cis. Second, the missense variant 1722 C $>$ A S574R, which affects the first base of exon 12a and may exert an effect by amino acid substitution or by interfering with normal splicing.

We also detected 22 nonsense mutations within the single base pair substitutions, of which $16(73 \%)$ have been described by previous investigators. In 13/22 (59\%) of the nonsense mutations the mutation occurs at a $\mathrm{CpG}$ dinucleotide and alters the primary base of the codon to a $\mathrm{T}$ (HGA>TGA), forming a termination signal.

Ten of the missense mutations affected bases potentially involved in splicing. These included five substitutions altering splice acceptors and one altering a splice donor. In addition we found two intronic deletions, which directly alter invariant splice site dinucleotides. 2991-4 del ATA changes the intron 17 acceptor site $A G>T G$, while $6641+1$ del $G$ appears to shift the intron 35 donor site 1 bp upstream. Three other potentially significant intronic sequence variations were found: $3113+3$ ins A, 4111-8 del GTT, and 288+5 $\mathrm{G}>\mathrm{C}$. None of these has been previously reported.

A second class of mutations, frame shifts, was detected in 21 patients. Fourteen were small deletions ranging from $1 \mathrm{bp}$ to $23 \mathrm{bp}$ in length. In this group only 499 del TGTT and 1756 del ACTA have been previously reported by other investigators. However, four other deletions had closely related mutations described in the literature, such as 3758 to 3762 del TCTAC related to 3759 to 3763 del CTACC, which was reported by Ars et al. ${ }^{22}$ All deletions were entirely exonic except $1-14$ to 7 del $21 \mathrm{bp}$ which deletes $14 \mathrm{bp}$ of non-coding sequence upstream of exon 1 together with $7 \mathrm{bp}$ of exon 1 , thereby removing the start codon.

Together, frameshift and nonsense mutations that would be expected to truncate the reading frame constituted 44 of the 89 mutations detected $(49.4 \%)$ in patients who fulfilled the NIH criteria. The distribution of these mutations appears to be evenly spread within the gene, with $27 / 44$ (61\%) predicted to terminate the protein in or upstream of the GRD.

A third class of mutations, insertions and deletions, was detected in 11 patients. Seven insertions were detected comprising five $1 \mathrm{bp}$ insertions and two $2 \mathrm{bp}$ insertions. None of these insertions has been previously described. Deletions detected included four in frame deletions; two removing a single amino acid and two removing two consecutive amino acids. Only 2970 del AAT $\Delta$ M991 and 7096 del $6 \Delta$ NF2366-7 have been previously reported. 
Table 2 Summary of NF1 mutations and sequence variations found. A total of 77 different potentially significant sequence variations were found, of which $57(74 \%)$ are novel

\begin{tabular}{|c|c|c|c|c|c|c|c|}
\hline Sample & NIH & Exon & Mutation & Type & Predicted effect & Accession & CpG \\
\hline D05-54 & NF & 1 & $1-14$ to 7 del $21 \mathrm{bp}$ & Frameshift & Truncated protein & & \\
\hline D04-07 & NF & 2 & 127 del CT V65X & Frameshift & Truncated protein & & \\
\hline D05-16 & NF & 3 & $271 \mathrm{G}>\mathrm{A}$ E91X & Nonsense & Truncated protein & & \\
\hline D05-69 & NF & $4 a$ & $311 \mathrm{~T}>\mathrm{A}$ L104X & Nonsense & Truncated protein & & \\
\hline D02-34 & $\mathrm{NF}$ & $4 \mathrm{~b}$ & 499 del TGTT D176X & Frameshift & Truncated protein & CD995532 & \\
\hline D02-65 & NF & $4 c$ & $597 / 8$ del 23 V207X & Frameshift & Truncated protein & & \\
\hline D05-53 & NF & 6 & $787 \mathrm{~A}>\mathrm{T} \mathrm{K} 263 \mathrm{X}$ & Nonsense & Truncated protein & & \\
\hline D02-16 & NF & 7 & 916 del G L316X & Frameshift & Truncated protein & & \\
\hline D02-49 & NF & 7 & $889-2 A>G$ & Splice acceptor & Truncated protein & CS991467 & \\
\hline D05-48 & NF & 9 & $1246 C>T$ R416X & Nonsense & Truncated protein & CM992366 & $\mathrm{Y}$ \\
\hline D02-11 & NF & $10 a$ & $1274 \mathrm{G}>\mathrm{A}$ W $425 \mathrm{X}$ & Nonsense & Truncated protein & CM000779 & \\
\hline D02-03 & NF & $10 a$ & 1318 C>T R440X & Nonsense & Truncated protein & CM950845 & $\mathrm{Y}$ \\
\hline D02-41 & NF & $10 a$ & $1318 \mathrm{C}>\mathrm{T}$ R440X & Nonsense & Truncated protein & CM950845 & Y \\
\hline D02-72 & NF & $10 a$ & $1318 \mathrm{C}>\mathrm{T}$ R440X & Nonsense & Truncated protein & CM950845 & $\mathrm{Y}$ \\
\hline D02-21 & NF & $10 \mathrm{~b}$ & $1527+1 G>C$ & Splice donor & Truncated protein & & \\
\hline D02-56 & NF & 11 & $1642-2 A>G$ & Splice acceptor & Truncated protein & & \\
\hline D02-23 & NF & $12 a$ & 1756 del ACTA $1603 X$ & Frameshift & Truncated protein & CD982825 & \\
\hline D05-11 & NF & 13 & 2124 del CT E715X & Frameshift & Truncated protein & & \\
\hline D05-43 & NF & 13 & 2034 ins C D699X & Frameshift & Truncated protein & & \\
\hline D05-10 & NF & 13 & $2180 \mathrm{C}>\mathrm{G}$ S727X & Nonsense & Truncated protein & & \\
\hline D02-12 & NF & 16 & $2851-2 A>G$ & Splice acceptor & Truncated protein & & \\
\hline D05-66 & NF & 18 & 2991-4 del ATA & Splice acceptor & Truncated protein & & \\
\hline D02-02 & NF & $19 a$ & 3163 C > T Q1055X & Nonsense & Truncated protein & & \\
\hline D05-74 & NF & 21 & 3528 del A L1183X & Frameshift & Truncated protein & & \\
\hline D05-45 & NF & 21 & 3546 del T L1 183X & Frameshift & Truncated protein & & \\
\hline D02-53 & NF & 22 & 3758 to 3762 del TCTAC F1261X & Frameshift & Truncated protein & & \\
\hline D05-73 & $\mathrm{NF}$ & 22 & $3721 \mathrm{C}>\mathrm{T}$ R1241X & Nonsense & Truncated protein & CM000799 & Y \\
\hline D05-78 & NF & 22 & $3721 \mathrm{C}>\mathrm{T}$ R1241X & Nonsense & Truncated protein & CM000799 & $\mathrm{Y}$ \\
\hline D02-09 & NF & 23.1 & 3916 C>T R1306X & Nonsense & Truncated protein & CM981381 & $\mathrm{Y}$ \\
\hline D02-17 & NF & 23.2 & 3982 ins A E1333X & Frameshift & Truncated protein & & \\
\hline D02-61 & NF & 23.2 & 4045 ins T S1373X & Frameshift & Truncated protein & & \\
\hline D04-08 & NF & 23.2 & 4095 ins TG & Frameshift & Truncated protein & & \\
\hline D05-90 & NF & 24 & 4265 C > A S1422X & Nonsense & Truncated protein & & \\
\hline D02-45 & NF & $27 a$ & $4537 C>T$ R1513X & Nonsense & Truncated protein & CM941093 & $\mathrm{Y}$ \\
\hline D05-87 & NF & $27 a$ & 4537 C > T R1513X & Nonsense & Truncated protein & CM941093 & $\mathrm{Y}$ \\
\hline D05-76 & NF & $27 a$ & $4515-1 \mathrm{G}>\mathrm{A}$ & Splice acceptor & Truncated protein & & \\
\hline D05-47 & NF & 28 & 4913 del TCTCT C1661X & Frameshift & Truncated protein & CD972357 & \\
\hline D05-57 & NF & 28 & 4950 ins A Y $1650 X$ & Frameshift & Truncated protein & & \\
\hline D05-62 & NF & 31 & 5898 del GA D1987X & Frameshift & Truncated protein & & \\
\hline D05-01 & NF & 31 & $5943+1 \mathrm{G}>\mathrm{T}$ & Splice donor & Truncated protein & & \\
\hline D02-31 & $\mathrm{NF}$ & 32 & $5944-1 \mathrm{G}>C$ & Splice acceptor & Truncated protein & & \\
\hline D05-04 & NF & 33 & 6181 del 8bp D2074X & Frameshift & Truncated protein & & \\
\hline D04-02 & NF & 35 & $6641+1$ del G & Splice donor & Truncated protein & & \\
\hline D04-05 & NF & 36 & $6709 C>T$ R2237X & Nonsense & Truncated protein & CM000815 & $\mathrm{Y}$ \\
\hline D05-70 & NF & 36 & $6709 C>T$ R2237X & Nonsense & Truncated protein & CM000815 & $\mathrm{Y}$ \\
\hline D02-62 & NF & 37 & $6792 C>A$ Y2264X & Nonsense & Truncated protein & CM981382 & \\
\hline D02-36 & NF & 37 & $6792 C>G$ Y2264X & Nonsense & Truncated protein & CM981382 & \\
\hline D05-65 & NF & 39 & 7096 ins A N2400X & Frameshift & Truncated protein & & \\
\hline D02-35 & NF & 40 & 7206 del CA C2405X & Frameshift & Truncated protein & & \\
\hline D05-09 & NF & 41 & 7285 C > T R2429X & Nonsense & Truncated protein & CM000818 & $\mathrm{Y}$ \\
\hline D02-22 & NF & 42 & 7427 ins TC S2502X & Frameshift & Truncated protein & & \\
\hline D02-30 & NF & 42 & 7486 C >T R2496X & Nonsense & Truncated protein & CM941096 & $\mathrm{Y}$ \\
\hline D05-55 & Q & $12 b$ & $1994 \mathrm{C}>\mathrm{T}$ S665F & Missense & Amino acid substitution & CM000785 & \\
\hline D02-40 & NF & $12 b$ & 1885 G > A G629R & Missense & Amino acid substitution & CM961026 & $\mathrm{Y}$ \\
\hline D02-68 & NF & $12 b$ & $1885 \mathrm{G}>\mathrm{A}$ G629R & Missense & Amino acid substitution & CM961026 & $\mathrm{Y}$ \\
\hline D02-78 & $Q$ & 16 & $2530 \mathrm{C}>\mathrm{T}$ L844F & Missense & Amino acid substitution & CM002379 & \\
\hline D02-38 & Q & 17 & 2970 del AAT $\triangle$ M991 & In frame deletion & Amino acid deletion & CD931025 & \\
\hline D05-72 & $Q$ & 26 & $4402 A>G$ S1 $468 G$ & Missense & Amino acid substitution & CM971049 & \\
\hline D02-06 & NF & 2 & $92 \mathrm{~A}>\mathrm{G} \mathrm{H} 31 \mathrm{R}$ & Missense & Amino acid substitution & & \\
\hline D02-54 & NF & 3 & $288+5 G>C$ & Intronic & Truncated protein & & \\
\hline D02-48 & NF & $4 a$ & $434 \mathrm{~T}>\mathrm{C} \mathrm{L145P}$ & Missense & Amino acid substitution & & \\
\hline D05-34 & Q & $4 b$ & $528 \mathrm{~T}>\mathrm{A}$ D 176E & Missense & Amino acid substitution & & \\
\hline D05-25 & NF & 6 & $846 \mathrm{G}>\mathrm{A}$ Q282Q & Same sense & Truncated protein & & \\
\hline D02-39 & NF & 6 & $846 \mathrm{G}>\mathrm{A}$ Q282Q & Same sense & Truncated protein & & \\
\hline D05-71 & NF & 7 & $1010 A>T$ E337V & Missense & Amino acid substitution & & \\
\hline D05-60 & NF & 7 & $970 \mathrm{~T}>\mathrm{C} C 324 \mathrm{R}$ & Missense & Amino acid substitution & & \\
\hline D02-18 & NF & $10 \mathrm{~b}$ & $1466 A>G \quad Y 489 C$ & Missense & Amino acid substitution & & \\
\hline D05-39 & NF & $10 \mathrm{~b}$ & $1466 A>G$ Y $489 C$ & Missense & Amino acid substitution & & \\
\hline D02-50 & $\mathrm{NF}$ & $10 b$ & $1466 A>G \quad Y 489 C$ & Missense & Amino acid substitution & & \\
\hline D02-10 & NF & $10 c$ & $1595 \mathrm{~T}>\mathrm{C}$ L532P & Missense & Amino acid substitution & & \\
\hline D02-15 & $\mathrm{NF}$ & $12 a$ & 1722 C $>$ A S574R & Missense & Truncated protein & & \\
\hline D05-64 & NF & 13 & $2040 \mathrm{C}>\mathrm{T}$ C $680 \mathrm{C}$ & Same sense & Truncated protein & & \\
\hline D05-58 & NF & 16 & $2514 C>G$ 1838M, $2531 \mathrm{~T}>\mathrm{C}$ L844P & Missense & Amino acid substitution & & \\
\hline D02-08 & NF & 16 & $2531 \mathrm{~T}>\mathrm{C}$ L844P & Missense & Amino acid substitution & & \\
\hline D04-12 & NF & 16 & $2617 \mathrm{C}>\mathrm{T}$ R873C & Missense & Amino acid substitution & & $\mathrm{Y}$ \\
\hline D02-05 & NF & 18 & $3113+3$ ins $A$ & Intronic & Truncated protein & & \\
\hline D02-52 & Q & $19 \mathrm{~b}$ & 3217 A $>G$ M1073V & Missense & Amino acid substitution & & \\
\hline D05-85 & Q & 21 & $3587 \mathrm{~T}>\mathrm{G}$ L1 196R & Missense & Amino acid substitution & & \\
\hline D05-42 & NF & 22 & $3826 C>G$ R1276G & Missense & Amino acid substitution & & $\mathrm{Y}$ \\
\hline D05-56 & NF & 22 & 3826 C > G R1276G & Missense & Amino acid substitution & & Y \\
\hline
\end{tabular}


Table 2 continued

\begin{tabular}{llllll}
\hline Sample & NIH & Exon & Mutation & Type & Predicted effect \\
\hline D05-19 & NF & 22 & 3827 G $>$ A R1276Q & Missense & Amino acid substitution \\
D05-27 & NF & 22 & 3827 G $>$ A R1 276Q & Missense & Amino acid substitution \\
D04-21 & NF & 24 & $4111-8$ del GTT & Intronic & Truncated protein \\
D05-33 & Q & 24 & 4255 A $>$ G K1419E & Missense & Amino acid substitution \\
D02-27 & NF & 25 & 4312 del GAA $\Delta$ E1 438 & In frame deletion & Amino acid deletion \\
D05-75 & NF & 28 & 4973 del 6 $\Delta$ YY1658-9 & In frame deletion & Amino acid deletion \\
D02-69 & NF & 28 & 5172 G $>$ A K1724K & Same sense & Truncated protein \\
D05-91 & NF & 29 & 5427 C $>$ T R1808R & Same sense & Truncated protein \\
D02-14 & NF & 39 & 7096 del 6 $\Delta$ NF2366-7 & In frame deletion & Amino acid deletion \\
\hline
\end{tabular}

$N F$, fulfils diagnostic criteria; $Q$, does not fulfil diagnostic criteria.

Four different conservative base substitutions were found in five unrelated, definitely affected patients. No other variations were detected in any of these patients and none of these conservative base substitutions were found in 260 normal chromosomes. Sequence variations 2040 C $>$ T C680C and $5427 \mathrm{C}>\mathrm{T}$ Rl808R were found in two patients in whom no other family members were available for testing at the time of writing. Another patient was found to have 5172 G>A K1724K and, on testing this patient's parents, the affected mother was found to carry the same variation while the father was found to be normal at this position. Finally, the variation 846 G>A Q282Q was found in two unrelated patients of eastern decent.

\section{Recurrent mutations}

Twenty of the 77 novel variations were detected in more than one unrelated individual or have been previously reported by other investigators (table 2). Ten variants were observed more than once in this study. All of these variations were single base pair substitutions and seven of them occurred at hypermutable $\mathrm{CpG}$ dinucleotides. In this study eight sequence variations were each found in two unrelated cases; 1885 G > A G629R, 2530 C > T L844F, 3721 C > T R1241X, 4537 $\mathrm{C}>\mathrm{T}$ R1513X, and $6709 \mathrm{C}>\mathrm{T}$ R2237X have also been previously reported by other investigators, whereas 3826C $>$ G R1276G， $3827 \mathrm{G}>\mathrm{A}$ R1276Q, and $846 \mathrm{G}>\mathrm{A}$ Q282Q have not. Two sequence variations were detected in three unrelated patients; the nonsense mutation $1318 \mathrm{C}>\mathrm{T}$ R440X has been previously reported while the missense variation $1466 \mathrm{~A}>\mathrm{G}$ Y489C is novel.
Two pairs of mutations were found to be very closely related, affecting a different base in the same codon. Codon 844 was alternatively mutated CTT > TTT (L844F), which was found twice and has been previously described, and CTT $>$ CCT (L844P), which is novel and was only found in one patient. Neither 3826C $>$ G R1276G, 3827 G $>$ A R 1276 Q have been previously described and both were found in two unrelated cases in this study. However two other mutations of this codon, $3826 \mathrm{C}>\mathrm{T}$ Rl276X, $3827 \mathrm{G}>\mathrm{C}$ Rl276Q have been previously reported. ${ }^{26}{ }^{27}$ Twenty other mutations found in this study were also found to have closely related mutations in the literature. Of particular note is $1466 \mathrm{~A}>\mathrm{G}$ Y489C, which was found in three cases in this study but has not been previously reported although Fahsold et $\mathrm{al}^{21}$ reported an identical mutation of an identical codon $2 \mathrm{bp}$ downstream of Y489C (1472 A $>$ G Y491C).

Although there seems to be a general spread of mutations found throughout the gene, we found no sequence changes in exons $1,5,15,20,27 \mathrm{~b}, 29,30,34,35,43,44,45,47,48$, or 49 .

\section{Polymorphisms}

Twenty sequence variations were found either in a normal subject or in an affected patient who already had a mutation characterised (table 3). Of these 14 had a rare allele frequency of less than 0.05 and eight were only found in a single subject in the entire study ( 167 subjects). Eight of the polymorphisms found were in the coding sequence and the remaining 12 all fell within 40 bases of an exon. All but three of the polymorphisms were single base pair substitutions, six being transversions and 11 being transitions, of which four

Table 3 Polymorphisms found during the mutation analysis process. Twenty such sequence variations were recorded in a normal subject or in an affected patient who had already had a mutation characterised

\begin{tabular}{|c|c|c|c|c|c|c|c|c|c|}
\hline \multirow{2}{*}{\multicolumn{2}{|c|}{ Exon Base }} & \multirow[b]{2}{*}{$\mathbf{P}$} & \multirow[b]{2}{*}{$\mathbf{Q}$} & \multirow{2}{*}{$\begin{array}{l}\text { Amino acid } \\
\text { change }\end{array}$} & \multirow[b]{2}{*}{$p$} & \multirow[b]{2}{*}{$q$} & \multicolumn{3}{|l|}{ Found with: } \\
\hline & & & & & & & Unaffected & Definite & Others \\
\hline 1 & $1-22 G>C$ & G & C & & 0.984 & 0.016 & $Y$ & 7486 C > T R2496X & $Y$ \\
\hline 2 & $61-4$ del T & 9Т & $8 \mathrm{~T}$ & & 0.996 & 0.004 & & $1642-2 A>G$ & \\
\hline 2 & $168 \mathrm{C}>\mathrm{T}$ & C & $\mathrm{T}$ & S56S & 0.959 & 0.041 & $Y$ & 4045 ins T S1373X & $Y$ \\
\hline $4 b$ & $528 \mathrm{~T}>\mathrm{A}$ & $\mathrm{T}$ & A & D176E & 0.996 & 0.004 & Y & & \\
\hline 5 & $702 \mathrm{~A}>\mathbf{G}$ & A & G & L234L & 0.728 & 0.364 & $\mathbf{Y}$ & many & $\mathbf{Y}$ \\
\hline 7 & 889-31 del ATTAT & & & & 0.996 & 0.004 & & 1885 G >A G629R & \\
\hline $10 \mathrm{~b}$ & $1393-32 \mathrm{C}>\mathrm{T}$ & C & $T$ & & 0.707 & 0.403 & $\mathbf{Y}$ & many & $\mathbf{Y}$ \\
\hline $10 c$ & 1528-29 del T & $8 \mathrm{~T}$ & $7 T$ & & 0.694 & 0.320 & $\mathbf{Y}$ & many & $\mathbf{Y}$ \\
\hline 11 & $1642-25 \mathrm{~T}>\mathrm{C}$ & $\mathrm{T}$ & C & & 0.973 & 0.027 & Y & 7096 del 6 & Y \\
\hline $12 a$ & $1810 \mathrm{~T}>\mathrm{C}$ & $\mathrm{T}$ & C & L604L & 0.993 & 0.007 & & $4255 \mathrm{~A}>\mathrm{G} \mathrm{K} 1419 \mathrm{E}$ & \\
\hline 16 & $2617 \mathrm{C}>\mathrm{T}$ & C & $\mathrm{T}$ & R873C & 0.996 & 0.004 & & $1-14$ to 7 del $21 \mathrm{bp}$ & \\
\hline 16 & $2531 G>A$ & G & A & G844G & 0.996 & 0.004 & & $1994 \mathrm{C}>\mathrm{T}$ S665F & \\
\hline 17 & $2851-16 \mathrm{~T}>\mathrm{C}$ & $\mathrm{T}$ & C & & 0.977 & 0.023 & Y & 3528 del A L1 183X & \\
\hline 22 & $3867 C>T$ & C & $\mathrm{T}$ & F1289F & 0.996 & 0.004 & & 1885 G>A G629R & \\
\hline 29 & 5546-19 T $>A$ & $\mathbf{T}$ & A & & 0.598 & 0.471 & $\mathbf{Y}$ & many & $\mathbf{Y}$ \\
\hline 32 & $6084+8 \mathrm{C}>\mathrm{G}$ & C & G & & 0.996 & 0.004 & & 1318 C > T R440X & \\
\hline 33 & $6173 \mathrm{C}>\mathrm{A}$ & C & $A$ & A2058D & 0.995 & 0.005 & & 6181del 8bp D2074X & \\
\hline 39 & $7126+37 \mathrm{G}>C$ & G & C & & 0.937 & 0.063 & $\mathbf{Y}$ & many & $\mathbf{Y}$ \\
\hline 42 & 7395-29 A $>$ G & A & G & & 0.666 & 0.356 & $\mathbf{Y}$ & many & $\mathbf{Y}$ \\
\hline 46 & $8050+20 \mathrm{G}>\mathrm{A}$ & G & $A$ & & 0.989 & 0.011 & Y & & \\
\hline
\end{tabular}


occurred at $\mathrm{CpG}$ dinucleotides. The remaining three polymorphisms comprised two deletions involving a single $\mathrm{T}$ and one deletion of $5 \mathrm{bp}$.

\section{DISCUSSION}

In this study we used direct sequencing and automated comparative sequence analysis to study 167 subjects including 91 unrelated definitely affected NF1 patients. Putative mutations were found in 81 of these affected patients $(89 \%)$, which means that our study achieved the highest recorded mutation detection rate using a single technique for this gene. Given that the current estimate of whole gene deletions in this disorder is approximately $10 \%$, this technique may therefore pick up all the remaining classes of mutations and potentially replaces all existing technologies, used either alone or together, with advantages of increased specificity and sensitivity associated with decreased cost and considerably reduced analysis times. When 96 well plates are used, an approximate cost for sequencing the entire NFl coding region for 1 patient is $£ 220$. Although commercial sequencing centres exist that may be able to match these prices the level of quality assurance, follow up, and guarantees needed for diagnostic laboratory purposes cannot be assured. A class of mutations that would not be detected with this assay are large gene deletions, as the test is not dosage sensitive. We plan to set up a multiplex ligation dependent probe amplification assay to look for whole exon and whole gene deletions in the patients in whom we have not found pathogenic mutations by sequence analysis.
Many new mutations have been identified in our study, with $74 \%$ being novel. This confirms that there are few recurrent mutations in NFl. While we find that mutations are evenly distributed along the NFl coding region, the scale of our present study allows further analysis of possible mutation hot spots and functional domains. The RasGAP activity of the central GAP related domain (GRD) and the structure of the GRD from neurofibromin have already been well characterised. ${ }^{128}$ Indeed clustering of mutations has been reported in this region, and much attention has since been concentrated on this area. The GRD is not shown to be a significant mutation hot spot in this study, with only $22 \%$ of mutations falling within this region. A second possible functional domain upstream of GRD has also been reported around exons 11-17. ${ }^{21}$ When our mutations were analysed by type and a weighted distribution was calculated for each exon, the number of missense and in frame deletions found to be clustered in this region was assessed visually and by means of a $\chi^{2}$ analysis on mutation percentage. This is shown in fig 2, confirming that exons 11-17 represent an important area of the NF1 protein. A similar cluster, which was termed a hot spot, was seen in the study of Fahsold et $\mathrm{al}^{21}$ although we detected more mutations in upstream exons. This region is known to be a domain rich in cysteine and serine domain, as defined by Izawa et al. This domain has three cysteine pairs suggestive of ATP binding as well as three potential cAMP-dependent protein kinase recognition sites, which are subject to phosphorylation by protein kinase. ${ }^{29}$ As abnormalities of CAMP signalling have been demonstrated in

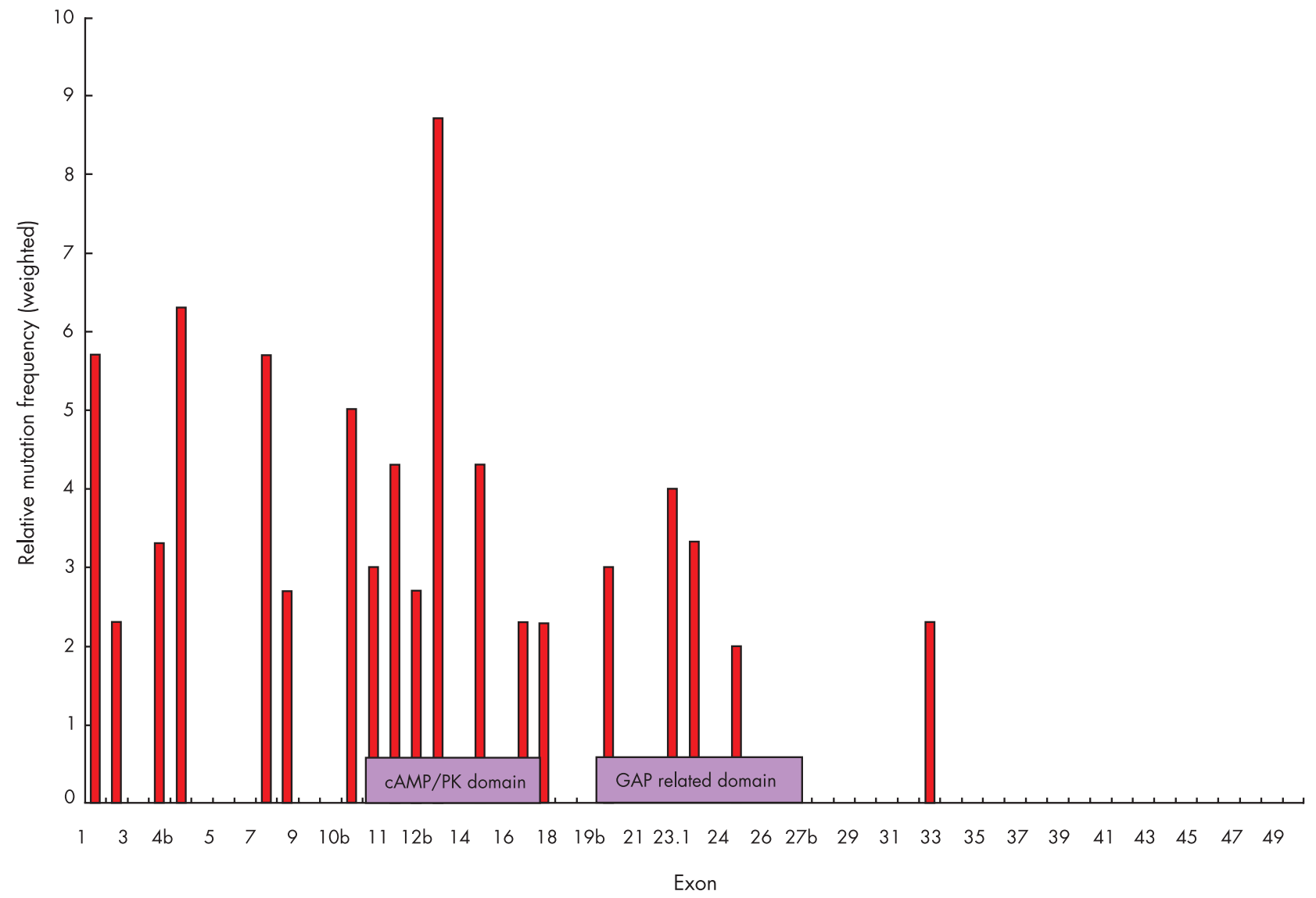

Figure 2 Weighted distribution of missense mutations and single base changes in the NF1 gene. The relative mutation frequency was calculated by dividing the number of missense or single base changes by the number of base pairs per exon. The value is then shown as a ratio between the exon specific mutation densities and the average mutation density for the whole gene (30/8473 bp). A clustering can be seen between exons $10-17$. Between exon group and number of mutations present $\chi^{2}=20.85$, df $3, p=0.0001$. Mutation rate is greatest between exons $1-20: \chi^{2}=11.74$, df 2 , $\mathrm{p}<0.005$, compared with exons 21-49: $\chi^{2}=9.1$, df $2, \mathrm{p}<0.01$. 
Drosophila neurofibromin mutations, it will be important to clarify further the link between cAMP and the Ras signalling pathway in NFl.

In previous studies that examined the effect of mutations at the RNA level it was found that $50 \%$ resulted in mutations causing splicing alterations. Splicing defects therefore appear to be the most common molecular defect in NF1. ${ }^{22}$ We have developed a functional splicing assay using a minigene system, ${ }^{30}$ which will allow us to clarify the significance of point mutations including missense or silent changes. One mutation found in this study has already been studied in this way. The 288+5 G>C was found to exclude exon 3 and further investigation showed that this aberrant splicing could be rescued by co-expression of an altered Ul-snRNA complementary to the mutation, proving that the mutation induces exon 3 skipping by preventing binding of Ul snRNA and thus causes the disease. This assay will be of great value in the analysis of mutations such as the previously described $6792 \mathrm{C}>\mathrm{G}$ (Y2264X), which affects splicing. ${ }^{22}$

In addition to clinical utility, the availability of a powerful mutation detection technique for the NFl gene will allow us to address two long standing questions in NFl. First, do phenotype-genotype correlations exist? This could be studied in a large number of patients where the whole gene is sequenced and attention paid to the type of mutation and clinical sign or symptom. Second, what is the contribution of the NFl gene to some of the rare related disorders, such as segmental NF, gastrointestinal NF, familial spinal NF and familial café au lait spots? For example, using this technique we have recently examined the rare syndrome Noonan-Neurofibromatosis where patients exhibit features of both disorders. Mutation analysis found NoonanNeurofibromatosis to be a subset of NFl in some cases, with mutations in the NFl gene. ${ }^{31}$ The high sensitivity of automated CSA makes analysis of the other rare related disorders feasible.

\section{ACKNOWLEDGEMENTS}

We thank the patients and their families for their cooperation and Dr S Goodburn for help with the statistical analysis.

\section{Authors' affiliations}

C Mattocks, D Baralle, P Tarpey, C ffrench-Constant, M Bobrow,

J Whittaker, Department of Medical Genetics, Box 134, Addenbrooke's Hospital, Cambridge, UK

C Mattocks, Salisbury Reference Laboratory, UK

P Tarpey, The Wellcome Trust Sanger Centre, Hinxton, UK

D Baralle, C ffrench-Constant, Department of Pathology, University of Cambridge, UK

Dr D Baralle is supported by Action Research and Prof. C ffrenchConstant is supported by the Wellcome Trust.

Correspondence to: Dr J Whittaker, Regional Genetics Laboratories, Molecular Genetics, Kefford House, Maris Lane, Trumpington, Cambridge, CB2 2FF; joanne.whittaker@addenbrookes.nhs.uk

Received 30 June 2003

Revised version received 20 November 2003

Accepted for publication 21 November 2003

\section{REFERENCES}

1 Upadhyaya M, Cooper D, eds. Neurofibromatosis type 1: from genotype to phenotype. Oxford: BIOS Scientific Publishers, 1998:65-88.

2 Riccardi VM. Neurofibromatosis: phenotype, natural history and pathogenesis, 2nd ed. Baltimore: John Hopkins University, 1992

3 Huson SM, Hughes RAC. The neurofibromatosis: a clinical and pathogenic overview. London: Chapman \& Hall, 1994.

4 Friedman JM, Birch PH. Type 1 Neurofibromatosis: a descriptive analysis of the disorder in 1728 patients. Am J Med Genet 1997;70:138-43.

5 Legius E, Marchuk DA, Hall BK, Anderson LB, Wallace MR, Collins FS, Glover TW. NF1-related locus on chromosome 15. Genomics 1992;13:1316-8.
6 Colman SD, Williams CA, Wallace MR. Benign neurofibromas in type 1 neurofibromatosis (NF1) show somatic deletions on NF1 gene. Nat Genet $1995 ; 11: 90-2$

7 Serra E, Puig S, Otero D, Gaona A, Kruyer H, Ars E, Estivill X, Lazaro C. Confirmation of a double hit model for the NFl gene in benign neurofibromas. Am J Hum Genet 1997:61:512-9.

8 Shannon KM, O'Connell P, Martin GA, Paderanga D, Olson K, Dinndorf P, McCormick F. Loss of normal NF1 allele from the bone marrow of children with type 1 neurofibromatosis and malignant myeloid disorders. N Engl J Med 1994:330:597-601.

9 Cawthorn RM, Weiss R, Xu G, Viskochil D, Culver M, Stevens J, Robertson M, Dunn D, Gesteland R, O'Connell P, White R. A major segment of the neurofibromatosis type 1 gene: cDNA sequence, genomic structure and point mutations. Cell 1990;62:193-201.

10 Viskochil D, Buchberg AM, Xu G, Cawthorn RM, Stevens J, Wolff RK, Culver M, Carey JC, Copeland NG, Jenkins NA, White R, O'Connell'P. Deletions and a translocation interrupt a cloned gene at the neurofibromatosis type 1 locus. Cell 1990;62:187-92.

11 Wallace MR, Marchuk DA, Anderson LB, Letcher R, Odeh HM, Saulino AM Fountain JW, Brereton A, Nicholson J, Mitchell AL, Brownstein BH, Collins FS. Type 1 neurofibromatosis gene: identification of a large transcript disrupted in three NF1 patients. Science 1990;249:181-6.

12 Daston MM, Scrable T, Nordlund N, Sturbaum AK, Nisse LM, Ratner N. The protein product of the neurofibromatosis type 1 gene is expressed at highest abundance in neurons, Schwann cells and oligodendrocytes. Neuron 1992;8:415-28.

13 Marchuk DA, Saulino AM, Tavakkol RL, Swaroop M, Wallace MR, Anderson LB, Mitchell AL, Gutmann DH, Boguski M, Collins F. cDNA cloning of the type 1 neurofibromatosis gene: complete sequence of the NF1 gene product. Genomics 1991;11:931-40.

14 Danglot G, Regnier V, Fauvet D, Vassal G, Kujas M, Bernheim A. Neurofibromatosis 1 (NF1) mRNAs expressed in the central nervous system are differentially spliced in the $5^{\prime}$ part of the gene. Hum Mol Genet 1995;4:915-20

15 Li Y, O'Connell P, Briedenbach HH, Cawthorn R, Stevens J, Xu G, Neil S, Robertson M, White R, Viskochil D. Genomic organization of the Neurofibromatosis 1 gene (NF1). Genomics 1995:25:9-18.

16 Xu G, O'Connell P, Viskochil D, Cawthorn R, Robertson M, Culver M, Dunn D, Stevens J, Gestelad R, White R, Weiss R. The neurofibromatosis type 1 gene encodes a protein related to GAP. Cell 1990;62:599-608.

17 Martin GA, Viskochil D, Bollag G, McCabe PC, Crosier WJ, Haubruck H, Conroy L, Clark R, O'Connell P, Cawthorn RM, et al. The GAP-related domain of the neurofibromatosis type 1 gene product interacts with ras p21. Cell 1990;63:843-9.

18 Guha A, Lau N, Huvar I, Gutmann D, Provias J, Pawson T, Boss G. ras-GTP levels are elevated in human NF1 peripheral nerve tumours. Oncogene $1996 ; 12: 507-13$

19 Upadhyaya M, Osborn MJ, Maynard J, Kim MR, Tamanoi F, Cooper DN Mutational and functional analysis of the neurofibromatosis type 1 (NF1) gene. Hum Genet 1997;99:88-92.

20 Costa RM, Federov NB, Kogan JH, Murphy GG, Stern J, Ohno M, Kucherlapati R, Jacks T, Silva AJ. Mechanism for the learning deficits in a mouse model of neurofibromatosis type 1. Nature 2002;415:526-30.

21 Fahsold R, Hoffmeyer S, Mischung C, Gille C, Ehlers C, Kucukceylan N, Abdel-Nour M, Gewies A, Peters H, Kaufmann D, Buske A, Tinschert S, Nurnberg P. Minor lesion mutational spectrum of the entire NF1 gene does no explain its high mutability but points to a functional domain upstream of the GAP-related domain. Am J Hum Genet 2000;66:790-818

22 Ars E, Serra E, Garcia J, Kruyer H, Gaona A, Lazaro C, Estivill X. Mutations affecting mRNA splicing are the most common molecular defects in patients with neurofibromatosis type 1. Hum Mol Genet 2000;9:237-47.

23 Messiaen LM, Callens T, Mortier G, Beysen D, Vandenbroucke I, Van Roy N, Speleman F, De Paepe A. Exhaustive mutation analysis of the NF1 gene allows identification of $95 \%$ of mutations and reveals a high frequency of unusual splicing defects. Hum Mutat 2000;15:541-55.

24 Mattocks C, Tarpey P, Bobrow M, Whittaker J. Comparative sequence analysis (CSA): a new sequence based method for the identification and characterisation of mutations in DNA. Hum Mutat 2000;16:437-43.

25 NIH. NIH Consensus Development Conference Statement (1998). Neurofibromatosis. Arch Neurol 1988;45:575-8.

26 Klose A, Robinson N, Gewies A, Kluwe L, Kaufmann D, Buske A, Tinschert S, Peters $\mathrm{H}$. Two novel mutations in exons $19 \mathrm{a}$ and 20 and a BsaBI [correction of Bsal] polymorphism in a newly characterized intron of the neurofibromatosis type 1 gene. Hum Genet 1998;102:367-71.

27 Heim RA, Kam-Morgan LN, Binnie CG, Corns DD, Cayouette MC, Farber RA, Aylsworth AS, Silverman LM, Luce MC. Distribution of 13 truncating mutations in the neurofibromatosis 1 gene. Hum Mol Genet 1995;4:975-81.

28 Scheffzek K, Ahmadian MR, Wiesmuller L, Kabsch W, Stege P, Schnitz F, Wittinghofer A. Structural analysis of the GAP-related domain from neurofibromin and its implications. EMBO J 1998;17:4313-27.

29 Izawa I, Tamanaki N, Soya H. Phosphorylation of neurofibromatosis type 1 gene product (neurofibromin) by cAMP-dependent protein kinase. FEBS Lett 1996:382:53-9.

30 Baralle M, Baralle D, De Conti L, Mattocks C, Whittaker J, Knezevich A, ffrench-Constant C, Baralle FE. Identification of a mutation that perturbs NFI gene splicing using genomic DNA samples and a minigene assay. J Med Genet 2003:40:220-2.

31 Baralle D, Mattocks C, Kalidas K, Elmslie F, Whittaker J, Lees M, Ragge N, Patton MA, Winter R, ffrench-Constant C. Different mutations in the NF1 gene are associated with Neurofibromatosis-Noonan syndrome (NFNS). Am J Med Genet 2003:119A:1-8. 